\title{
Automatic Modulation Recognition of Communication Signals
}

\author{
Yun Lin ${ }^{1,2}$ and Chunguang $\mathrm{Ma}^{1}$ \\ ${ }^{1}$ College of Computer Science and Technology \\ ${ }^{2}$ College of Information and Communication Engineering \\ Harbin Engineering University, P.R. China \\ linyun@hrbeu.edu.cn
}

\begin{abstract}
Modulation identification of communication signals is an important content of the electronic countermeasure procedure and a hot topic in the research field of signal processing. Generally speaking, the essential problem of communication signals' recognition is a pattern recognition problem. In these paper, Firstly, the feasibility of the improved method to distinguish between analog modulation signals and digital modulation signals based on Haar Wavelet Transform(HWT) is demonstrated. Secondly, a identification method of analog modulation signals based on the higher order moment of envelope and the spectrum symmetry is presented. Thirdly, an identification method of digital modulation signals using high-order cumulants is suggested. Finally, an experiments is designed to prove the validity of the research theory. The final result shows that the recognition method in this paper has a well prospect in communication signals' recognition.
\end{abstract}

Keywords: Communication signal's recognition; Wavelet transform; higher-order moment of envelope; High-order cumulants;

\section{Introduction}

There are so many communication signals all available with different types of modulation and different frequencies. It is necessary to identify and closely monitor these signals for few applications. In military, there are many applications such as surveillance, electronic warfare and threat analysis. One of the important characteristics is the signal modulation type which describes how the information is being carried in the signal. In civilian, there are many applications such as signal confirmation, interference identification, monitoring and spectrum management.

In this paper, the modulation recognition of the signals include AM, FM, DSB, LSB, USB, 2ASK/BPSK, 4ASK, QPSK, 8PSK, 16QAM, 32QAM are researched, Firstly, as an effective symbol rate estimation method, wavelet transform theory has been widely used method in distinguishing between analog modulation signals and digital modulation signals[1-3] and a improved method has been proposed to solve some problems in common methods. Secondly, after the signal prior information whether it is analog modulation or digital modulation is acquired, the higher order moment of envelope $J$ and the spectrum symmetry $P$ can be used to identify the analog modulation signals [4-5] and the high-order cumulants theory can be used to identify the digital modulation signals[68]. In the last section of the paper, the effectiveness of the approach is proved by a scientific experiment based on MATLAB software. 


\section{Proposed Modulation Recognition Procedure}

\subsection{Communication Signals Model}

The waveform of analog modulation signals with noise pollution can be expressed as

$$
r(t)=m(t) \cos \left[2 \pi f_{c} t+\phi(t)+\theta\right]+n(t)
$$

And the digital modulation signals with noise pollution can be expressed as

$$
r(t)=\sum_{k} a_{k} \sqrt{E} p\left(t-k T_{s}\right) \exp \left(j 2 \pi f_{c} t+j \theta_{c}\right)+n(t), k=1,2, \ldots N
$$

Among them, $m(t)$ is the amplitude of the signal, $f_{c}$ is the carrier frequency, $\phi(t)$ is the modulated phase, $\theta$ is the initial phase, $\mathrm{N}$ is the length of element sending sequence, $a_{k}$ is the sequence of transmitting symbol, $E$ is the symbol energy of transmitting codes, $p(t)$ is the waveform of transmitting symbols, $T_{s}$ is the code width, $\theta_{c}$ is the carrier phase, $n(t)$ is Gaussian white noise with zero mean, and the signals and noise $n(t)$ are independent. Suppose carrier frequency, phase and timing are all synchronized, after the conversion, the digital modulation signals can be expressed as

$$
r(t)=\sum_{k} \sqrt{E} a_{k} p\left(t-k T_{s}\right) \exp \left(j \theta_{c}\right)+n(t)
$$

\subsection{Recognition between Analog Modulation Signals and Digital Modulation Signals}

K.C.Ho first proposed a method based on wavelet transform to estimate the symbol rate[1].This is a linear transformation method. In order to properly characterize the characteristics when the code of digital communication signal is switching, Haar wavelet was used as the mother wavelet function. QAM, PSK and FSK signals in analytical form of intermediate frequency can be expressed as:

$$
s(k)= \begin{cases}\sqrt{s_{i}} e^{j\left(\varphi_{i}+\omega_{i} k\right)} e^{j\left(\omega_{c} k+\theta_{c}\right)} & (i-1) T \leq k \leq i T \\ \sqrt{s_{i+1}} e^{j\left(\varphi_{i+1}+\omega_{i+1} k\right)} e^{j\left(\omega_{c} k+\theta_{c}\right)} & i T \leq k \leq(i+1) T\end{cases}
$$

Here, $\sqrt{s_{i}}, \phi_{i}$ and $\omega_{i}$ are respectively the information of amplitude, phase and frequency, $\omega_{c}$ and $\theta_{c}$ are respectively the frequency and initial phase of the carrier wave after the signal was shifted to intermediate frequency. The amplitude of PSK and FSK signals remain unchanged,only the frequency of FSK signals will change, and the phase of QAM and PSK signals will change in the code switching.

If the wavelet transform integral interval is in a code period, the steady-state value of WT can be gotten as follow:

$$
\left|W T_{\tilde{s}}(a, n)\right|=2 \sqrt{\frac{1}{a}}\left|\frac{\sin ^{2}\left[\left(\omega_{c}+\omega_{i}\right) a / 4\right.}{\sin \left[\left(\omega_{c}+\omega_{i}\right) / 2\right.}\right|,(i-1) T+\frac{a}{2} \leq n \leq i T-\frac{a}{2}
$$

If the integral interval spans across two code periods, the value of WT when $n=i T$ can be gotten as follow:

$$
\left|W T_{\tilde{s}}(a, n)\right|=2 \sqrt{\frac{1}{a}}\left|\frac{\sin \left(\omega_{c} a / 4\right) \sin \left(\omega_{c} a / 4+\alpha / 2\right)}{\sin \left(\omega_{c} / 2\right)}\right|, n=i T
$$

Where $\alpha=\phi_{i+1}-\phi_{i}$ is the phase jump of adjacent code cells and $\tilde{s}=s /|s|$.

According to the formula of the HWT, the frequency of digital modulation signal is reflected by the steady-state value in the same element cycle, and the phase jump between adjacent element is reflected by the transient value. It can be deduced that the Haar 
Wavelet Transform have good transient detection capability. So the result of HWT and codes of signals are shown in a same periodic property. The method uses wavelet detection ability of transient to detect the switching point of the adjacent symbols, then estimate the symbol rate through Fourier transform from the pulse sequence which represents the mutations. The method by detecting the presence of symbol rate to distinguish between analog modulation signals and digital modulation signal has been widely used at present.

However, the steady-state value and the transient value of HWT are both close to zero value when $\omega_{c} a \approx 4 n \pi, n=1,2, \ldots$, the peak sequence caused by phase jump is not obvious, the code period cannot be gotten, this kind of situation is called the Carrier Frequency Blind Spots ${ }^{[9]}$. And there are difficulties in the setting of wavelet scales. Y.T.Chan proposed an improved method to solve the problems of blind spots and wavelet scales difficulties by superposing the results of multi-scale wavelet transform ${ }^{[2]}$, but it did not resolve the problem essentially, the amount of calculation was greatly increased, the anti-noise ability of the method is poor and the improvement of effect is limited.

In this paper, the frequency of the signal is firstly shifted to the baseband, when $\omega_{c} \approx 0$, the steady-state value and the transient value of HWT can be expressed as follows:

$$
\begin{aligned}
& \left|W T_{s}(a, n)\right| \approx \sqrt{a}\left|\sin \left(\omega_{c} a / 4\right)\right| \approx 0, \quad(i-1) T+\frac{a}{2} \leq n \leq i T-\frac{a}{2} \\
& \left|W T_{s}(a, n)\right| \approx \frac{2|\sin (\alpha / 2)|}{\sqrt{a}}\left(\frac{a}{2}-|n-i T|\right), \quad i T-\frac{a}{2} \leq n \leq i T+\frac{a}{2}
\end{aligned}
$$

As we can see from formula (2-7) and (2-8), the results have nothing to do with carrier frequency $\omega_{c}$, so there is no invalid wavelet scales problem, the carrier frequency blind spots problem can be solved, then scale 16, the most effective scale of wavelet transform supported in literature ${ }^{[9]}$, is chosen.
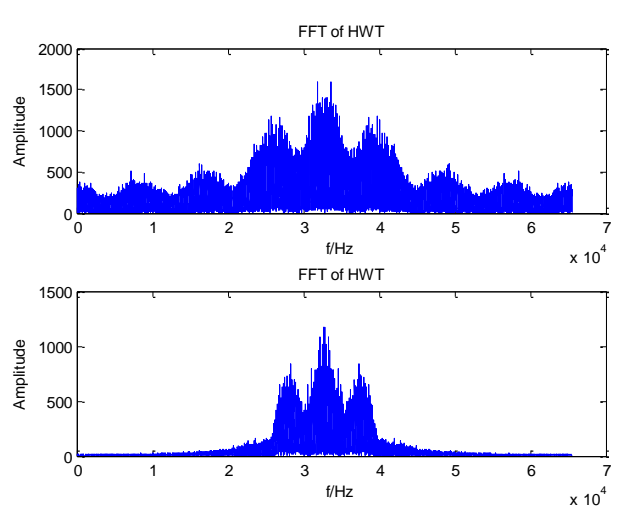

Figure 1(a). Result of AM before and after DDC
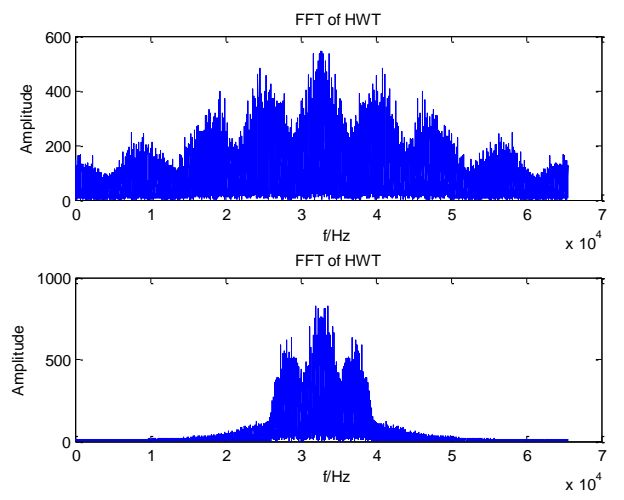

Figure 1(b). Result of FM before and after DDC 

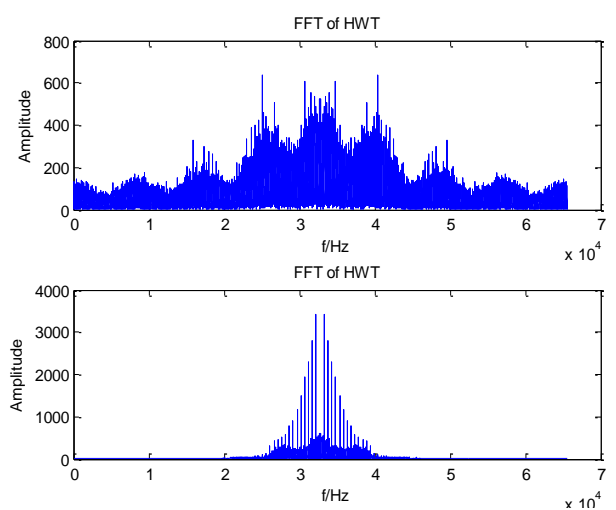

Figure 2(a). Result of BPSK before and after DDC
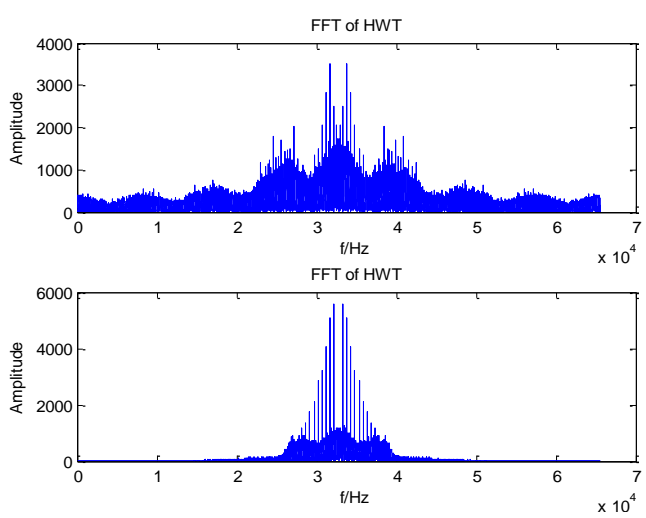

Figure 2(b). Result of 16QAM before and after DDC

As the examples shown in Fig.1 and Fig.2, when SNR equal to $1 \mathrm{~dB}$, there is no obviously spectral line found in the spectrum of the AM and FM signals' HWT results before and after DDC, in other words, there is no information of symbol rate can be found from analog modulation signals. However, the result of HWT of the BPSK and 16QAM signals are just the opposite. And from the figures of digital modulation signals, the spectral lines in the HWT results after DDC are more obvious and more well-ordered, so the detection of spectral lines will be easier in low signal-to-noise ratio.

It has been confirmed that the improved method gets higher stability and achieves a better effect. Through measuring the interval between the peaks several times and getting the variance of the intervals, the signal can be estimated to be a analog modulation signal or a digital modulation signal.

\subsection{Recognition of Analog Modulation Signals}

The common analog modulation signals in practical communication systems are AM, FM, LSB, USB, DSB signals. A.K.Nandi proposed a method using the characteristic parameter $\gamma_{\max }, \sigma_{a p}, \sigma_{d p}, P$ to classify signals AM, DSB, LSB, USB, FM, VSB, AMFM. It is a common method widely used to classify the analog modulation signals, but the unwrapped phase information which is is sensitive to the noise needs to be extracted to calculate parameter $\sigma_{a p}$ and $\sigma_{d p}$, this paper points out that when the signal-to-noise ratio is lower than $10 \mathrm{db}$, the recognition accuracy rate is very low. And the method can be functional only when some advanced information of the received signal has been known. In this paper, a effective method using the higher order moment of envelope $J$ and the spectrum symmetry $P$ is proposed to classify signals AM, FM, LSB, USB, DSB in the condition of low signal-to-noise ratio.

The received signal $r(t)$ is defined by

$$
r(t)=s(t)+n(t)=m(t) \cos [\phi(t)]+n(t)
$$

where $s(t)$ is the modulated signal, $n(t)$ is a Gaussian white noise variance to $\sigma_{n}^{2}$. $s(t)$ and $n(t)$ are independent of each other. $A(t)$ and $\phi(t)$ are respectively the information of amplitude and phase of the modulated signal.

The square of the amplitude envelope $\zeta(t)$ of the received signal $r(t)$ can be defined by

where

$$
\zeta^{2}(t)=r^{2}(t)+\hat{r}^{2}(t)=m^{2}(t)+2 m(t) a(t)+c^{2}(t)
$$

$$
a(t)=n(t) \cos [\phi(t)]+\hat{n}(t) \sin [\phi(t)]
$$




$$
c^{2}(t)=n^{2}(t)+\hat{n}^{2}(t)
$$

where $\hat{r}(t)$ is the result of the Hilbert transform of $r(t)$ and $\hat{n}(t)$ is the result of the Hilbert transform of $n(t)$. Because of the independence between $s(t)$ and $n(t)$, second order moments $m_{2}$ and fourth-order moments $m_{4}$ can be written as follows:

$$
\begin{gathered}
m_{2}=E\left[\zeta^{2}(t)\right]=E\left[A^{2}(t)+2 A(t) a(t)+c^{2}(t)\right]=E\left[A^{2}(t)\right]+2 \sigma_{n}^{2} \\
m_{4}=E\left[\zeta^{4}(t)\right]=E\left\{\left[A^{2}(t)+2 A(t) a(t)+c^{2}(t)\right]^{2}\right\}=E\left[A^{4}(t)\right]+8 \sigma_{n}^{2} E\left[A^{2}(t)\right]+8 \sigma_{n}^{4}
\end{gathered}
$$

According to the formula above, it can be deduced that

$$
m_{4}-2\left(m_{2}\right)^{2}=E\left[\zeta^{4}(t)\right]-2 E^{2}\left[\zeta^{2}(t)\right]=E\left[A^{4}(t)\right]-2 E^{2}\left[A^{2}(t)\right]
$$

It is easily known that the the influence of the noise is eliminated, the normalization is processed to the formula(2-15) by four times the square of the power of $s(t)$, and then define the parameter $J$ as follow

$$
J=\frac{m_{4}-2\left(m_{2}\right)^{2}}{4 S^{2}}=\frac{E\left[A^{4}(t)\right]-2 E^{2}\left[A^{2}(t)\right]}{4 S^{2}}
$$

\begin{tabular}{|c|c|c|c|c|}
\hline & theoretical formula & $E\left[A^{4}(t)\right]$ & $E\left[A^{2}(t)\right]$ & $J$ \\
\hline $\mathrm{AM}$ & $s(t)=[K+m(t)] A \cos \left(2 \pi f_{c} t+\theta\right)$ & $A^{4}\left(K^{4}+3 \sigma_{m}^{4}+6 K^{2} \sigma_{m}^{2}\right)$ & $A^{2}\left(K^{2}+\sigma_{m}^{2}\right)$ & $1-2 K^{4} /\left(K^{2}+\sigma_{m}^{2}\right)^{2}$ \\
\hline FM & $s(t)=A \cos [\phi(t)]$ & $A^{4}$ & $A^{2}$ & 1 \\
\hline DSB & $s(t)=A m(t) \cos \left(2 \pi f_{c} t+\theta\right)$ & $3 A^{4} \sigma_{m}^{4}$ & $A^{2} \sigma_{m}^{2}$ & 0 \\
\hline SSB & $s(t)=A[m(t)+\hat{m}(t)] \cos \left(2 \pi f_{c} t+\theta\right)$ & $8 A^{4} \sigma_{m}^{4}$ & $2 A^{2} \sigma_{m}^{2}$ & -1 \\
\hline
\end{tabular}

The value of characteristic parameter $J$ for signals AM, DSB, SSB, FM are obviously different, therefore $J$ can be used in classify those signals. The theoretical $J$ value of reference signals are shown in the table I below.

Table 1. Parameters $\mathrm{J}$ of Analog Modulation Signals

And the simulation results as we can see in figure 3 based on MATLAB show that the parameter is effective to classify the reference signals, where the baseband signal $m(t)$ is a zero mean Gaussian white noise after the low pass filter and $n(t)$ is another independent zero-mean Gaussian white noise. Among them, $\sigma_{m}^{2}=0.5, A=1, K=2, f_{c}=10 \mathrm{KHz}, f_{s}=8 \times f_{c}$, the length of the signal data $N_{s}=2048$. 


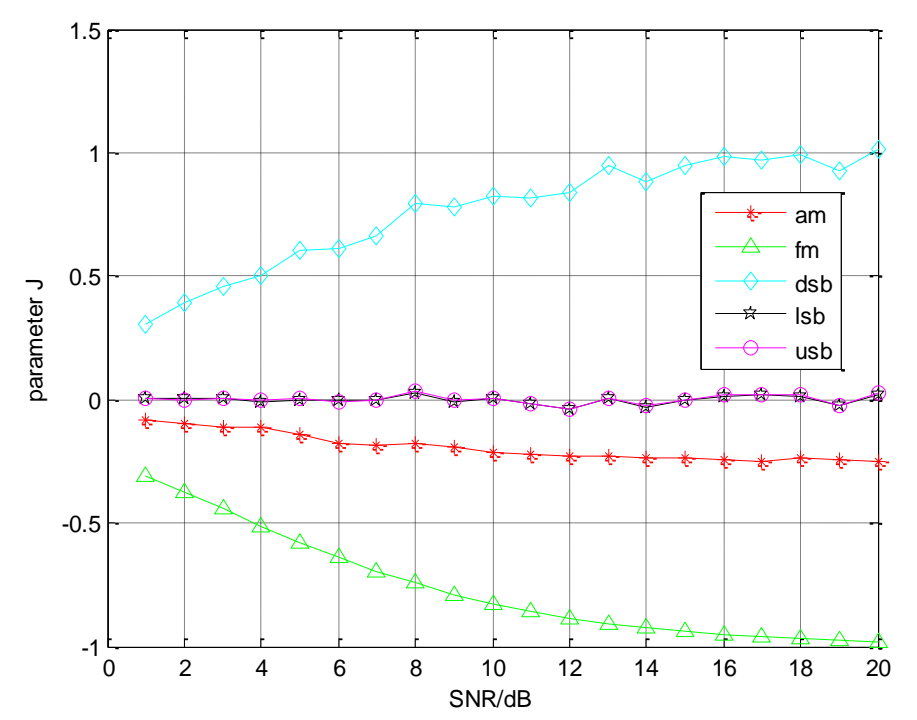

Figure 3. Parameter J of Analog Modulation Signals

The results of simulation which evaluates the parameter $J$ of analog modulation signals under different signal-to-noise ratios are summarised in Figure 3, it is clear that the $J$ values of the reference analog modulation signals are different, so the threshold value can be configured as Table 2 below.

Table 2. The Threshold Value of Parameter $\mathbf{J}$

\begin{tabular}{|l|l|}
\hline signal & J \\
\hline AM & $-0.4<J<-0.1$ \\
\hline FM & $-1<J<-0.4$ \\
\hline DSB & $J>0.2$ \\
\hline SSB & $-0.1<J<0.2$ \\
\hline
\end{tabular}

Due to the asymmetry of the signal spectrum of SSB signals, the spectrum symmetry $P$ can be used to classify LSB and USB signals , $P$ is defined as

$$
\begin{gathered}
P=\frac{P_{L}-P_{U}}{P_{L}+P_{U}} \\
P_{L}=\sum_{i=1}^{f_{c n}}\left|F_{r}(i)\right|^{2} \\
P_{U}=\sum_{i=1}^{f_{c n}}\left|F_{r}\left(i+f_{c n}+1\right)\right|^{2}
\end{gathered}
$$

where $F_{r}(i)=F F T[r(n)]$ is the Fourier transform of the received signal, and $f_{c n}=\frac{f_{c} \times N_{s}}{f_{s}}-1$. In ideal condition without noises, the spectrum of LSB signal only have the lower sideband $\left(P_{U}=0, P=1\right.$ ) and the spectrum of USB signal only have the upper sideband $\left(P_{L}=0, P=-1\right)$. 


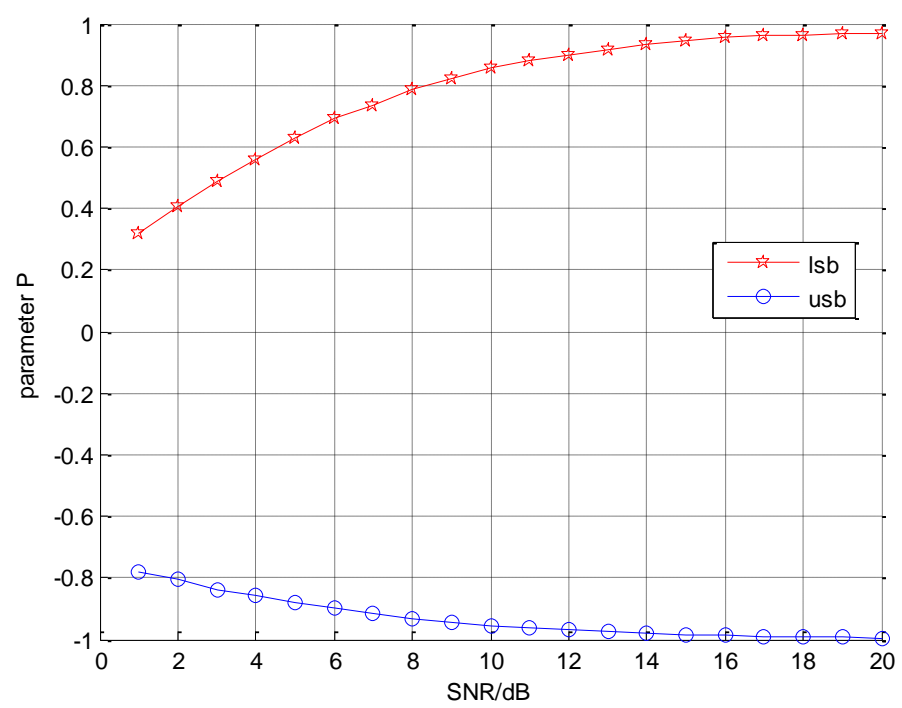

Figure 4. Parameter P of LSB and USB Signals

From Figure 4, it is clear that LSB and USB signals can be easily classified by parameter $P$, and the threshold value can be set to 0 .

\subsection{Recognition of Digital Modulation Signals}

Due to the half invariance of cumulants, there is

$$
\operatorname{Cum}[r(t)]=\operatorname{Cum}[s(t)]+\operatorname{Cum}[n(t)]
$$

where $r(t)=s(t)+n(t)$, and $n(t)$ is a Gaussian noise.

Because the result of the cumulants of the Gaussian noise is constantly zero when the cumulants order is in excess of second-order, The cumulants of received signal can be written as

$$
\operatorname{Cum}[r(t)]=\operatorname{Cum}[s(t)]
$$

So the influence of Gaussian noise can be eliminated when the non-Gaussian signals containing Gaussian noise are transformed to the cumulants domain. High-order cumulants of signals has very good noise resistance performance, so communication signals modulation recognition algorithm based on high-order cumulants has received the widespread attention.

Yu Han uses fourth-order and sixth-order cumulants to classify signals BPSK, QPSK, OQPSK, $\pi / 4$ DQPSK, 8PSK, 16QAM, 16APK, 64QAM. Huang Y and Lei J make use of extracting features of higher order cumulants to recognize BPSK, QPSK, 8PSK, 16QAM, 64QAM, 4ASK, 2FSK, 4FSK signals. LV Xin-zheng utilizes second-order, fourth-order, sixth-order cumulants to reach the signals classification of 2ASK, 4ASK, 4PSK, 2FSK, 4FSK. In this paper, the recognition of digital modulation signals including 2ASK, 4ASK, BPSK, QPSK, 8PSK, 16QAM signals using high-order cumulants has been researched.

For a stationary complex random process $X(k)$ with zero-mean, the high-order cumulants can be defined as the follows:

$$
\begin{gathered}
C_{20}=\operatorname{Cum}\left[X(k) X\left(k+l_{1}\right)\right] \\
C_{21}=\operatorname{Cum}\left[X(k) X^{*}\left(k+l_{1}\right)\right] \\
C_{40}=\operatorname{Cum}\left[X(k) X\left(k+l_{1}\right) X\left(k+l_{2}\right) X\left(k+l_{3}\right)\right] \\
C_{41}=\operatorname{Cum}\left[X(k) X\left(k+l_{1}\right) X\left(k+l_{2}\right) X^{*}\left(k+l_{3}\right)\right] \\
C_{42}=\operatorname{Cum}\left[X(k) X\left(k+l_{1}\right) X^{*}\left(k+l_{2}\right) X^{*}\left(k+l_{3}\right)\right]
\end{gathered}
$$




$$
\begin{gathered}
C_{60}=\operatorname{Cum}\left[X(k) X\left(k+l_{1}\right) X\left(k+l_{2}\right) X\left(k+l_{3}\right) X\left(k+l_{4}\right) X\left(k+l_{5}\right)\right] \\
C_{63}=\operatorname{Cum}\left[X(k) X\left(k+l_{1}\right) X\left(k+l_{2}\right) X^{*}\left(k+l_{3}\right) X^{*}\left(k+l_{4}\right) X^{*}\left(k+l_{5}\right)\right]
\end{gathered}
$$

when $l_{1}=l_{2}=l_{3}=\ldots=0$, the high-order cumulants can be simplified as follows:

$$
\begin{gathered}
C_{40}=M_{40}-3 M_{20}^{2} \\
C_{41}=M_{41}-3 M_{21} M_{20} \\
C_{42}=M_{42}-\left|M_{20}\right|^{2}-2 M_{21}^{2} \\
C_{60}=M_{60}-15 M_{20} M_{40}+30 M_{20}^{3} \\
C_{63}=M_{63}-6 M_{20} M_{41}-9 M_{42} M_{21}+18 M_{20}^{2} M_{21}+12 M_{21}^{3}
\end{gathered}
$$

where $M_{p q}=E\left\{X(k)^{p-q}\left[X^{*}(k)\right]^{q}\right\}$. The results of high-order cumulants of digital modulation signals are shown in the table as follow

Table 3. High-Order Cumulants of Digital Signals

\begin{tabular}{lcrrrrrr}
\hline & $C_{20}$ & $C_{21}$ & $C_{40}$ & $C_{41}$ & $C_{42}$ & \multicolumn{1}{c}{$C_{60}$} & \multicolumn{1}{c}{$C_{63}$} \\
\hline 2ASK & $E$ & $E$ & $-2 E^{2}$ & $-2 E^{2}$ & $-2 E^{2}$ & $16 E^{3}$ & $16 E^{3}$ \\
4ASK & $E$ & $E$ & $-1.36 E^{2}$ & $-1.36 E^{2}$ & $-1.36 E^{2}$ & $8.32 E^{3}$ & $8.32 E^{3}$ \\
2PSK & $E$ & $E$ & $2 E^{2}$ & $2 E^{2}$ & $2 E^{2}$ & $16 E^{3}$ & $16 E^{3}$ \\
4PSK & 0 & $E$ & $E^{2}$ & 0 & $E^{2}$ & 0 & $4 E^{3}$ \\
8PSK & 0 & $E$ & 0 & 0 & $E^{2}$ & 0 & $4 E^{3}$ \\
16QAM & 0 & $E$ & $0.68 E^{2}$ & 0 & $0.68 E^{2}$ & 0 & $2.08 E^{3}$ \\
\hline
\end{tabular}

From Table 3, it can be seen that 2ASK and 2PSK signals' cumulants of each order are the same, so it could not be used to distinguish 2ASK and 2PSK signals by establishing the parameters of its higher order cumulants. Known from the reference ${ }^{[10]}$, envelope characteristic of signals can be used to make the distinctions between 2ASK and 2PSK signals, two signals are classified as the same class in the following analysis of recognition.

So the classification feature vector is defined by $f_{r}=\left[f_{1}, f_{2}, f_{3}\right]$ to identify those signals.

Where $f_{1}=C_{40} / C_{42}, f_{2}=C_{41} / C_{42}, f_{3}=C_{63}^{2} / C_{42}^{3}$. As shown in the table I, for signals of different modulation types, the values of the classification feature vector $f_{r}$ are different as follows

$$
f_{r}=\left[f_{1}, f_{2}, f_{3}\right]=\left\{\begin{array}{cc}
{[1,1,32]} & 2 A S K / 2 P S K \\
{[1,0,16]} & 4 P S K \\
{[1,1,27.52]} & 4 A S K \\
{[0,0,16]} & 8 P S K \\
{[1,0,13.76]} & 16 Q A M
\end{array}\right.
$$




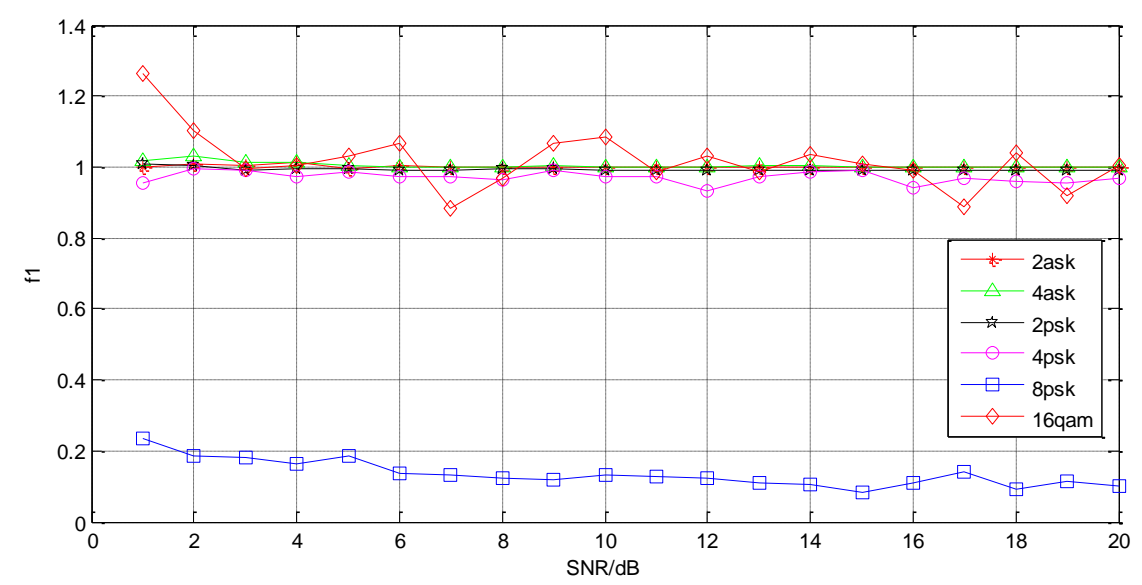

Figure 5. Parameter $f_{1}$ of the Reference Digital Modulation Signals

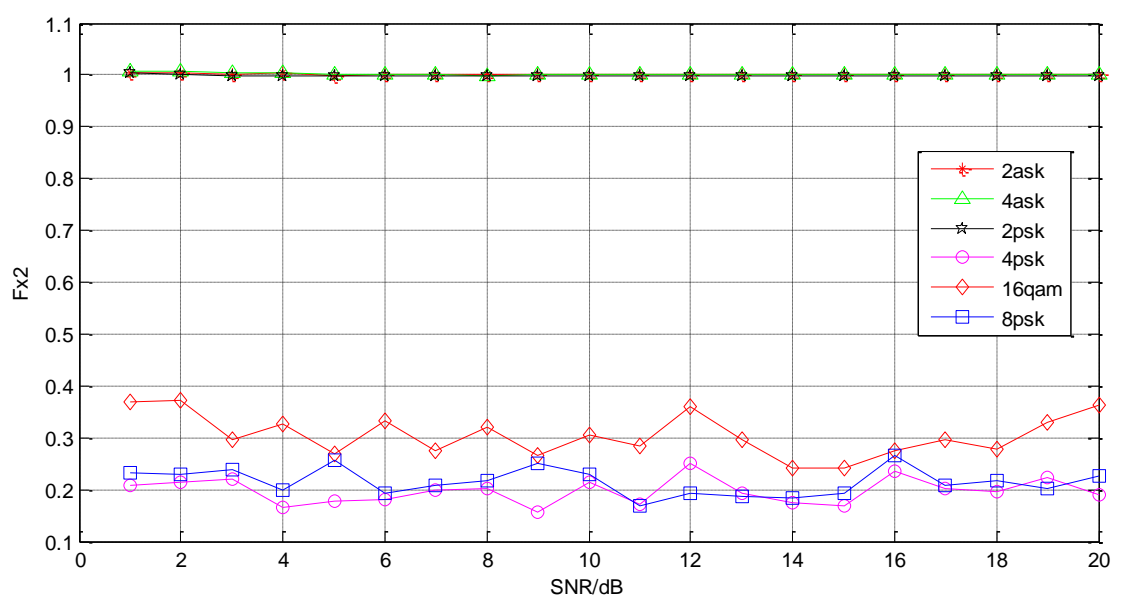

Figure 6. Parameter $f_{2}$ of the Reference Digital Modulation Signals

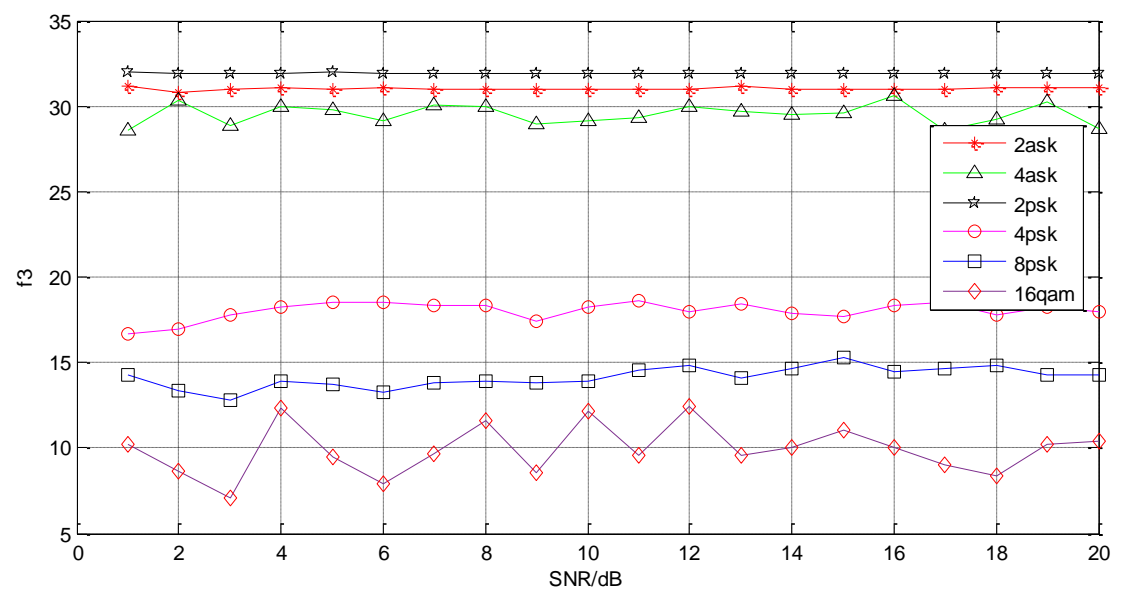

Figure 7. Parameter $f_{3}$ of the Reference Digital Modulation Signals 


\section{Results of Computer Simulations}

In this thesis, in order to prove the effectiveness and accuracy of the method mentioned in the paper, supposed the baseband signals of digital modulation signals are random codes and baseband signals of analog modulation signals are Gaussian white noise through a low-pass filter, supposed $f_{b}=2 \mathrm{KHz}, f_{c}=16 \times f_{b}, f_{s}=8 \times f_{c}, A=1$, $N=2048$. the results of computer simulations are shown below:

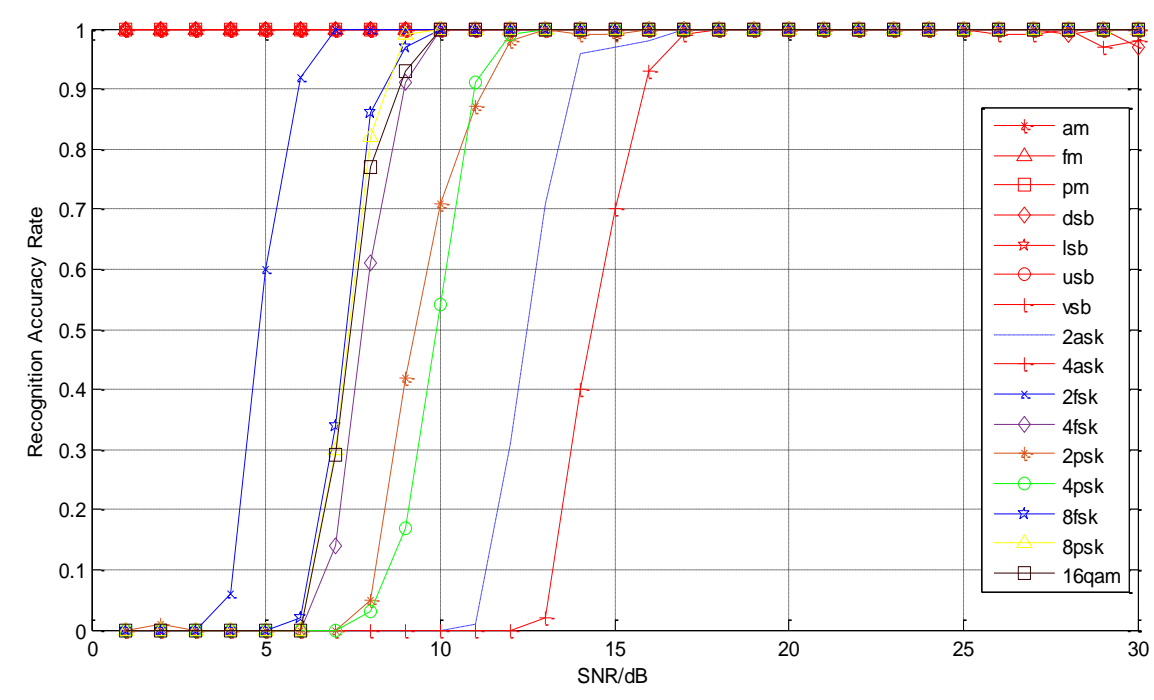

Figure 8. Accuracy Rate of Recognition between Analog and Digital Modulation Signals before DDC

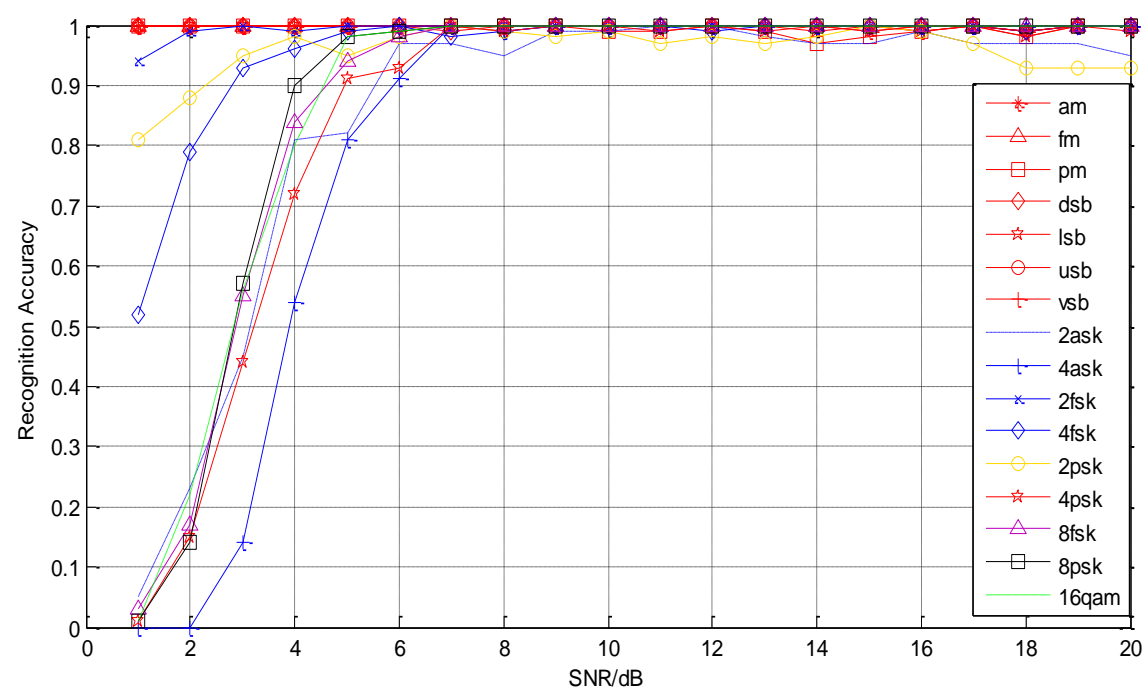

\section{Figure 9. Accuracy Rate of Recognition between Analog and Digital Modulation Signals after DDC}

As shown in Figure 8 and Figure 9, almost all common types of communication signals are researched to compare the performance of new method and traditional method. The method by detecting the presence of symbol rate to distinguish between analog modulation signals and digital modulation signal is effective, and the result of signals after DDC has much better performance than the result before DDC. It can be easily 
found that the averaged recognition rate of the improved method is as high as $90 \%$ when $S N R \geq 6 \mathrm{~dB}$, there is about $10 \mathrm{~dB}$ improvement compared to the traditional method.

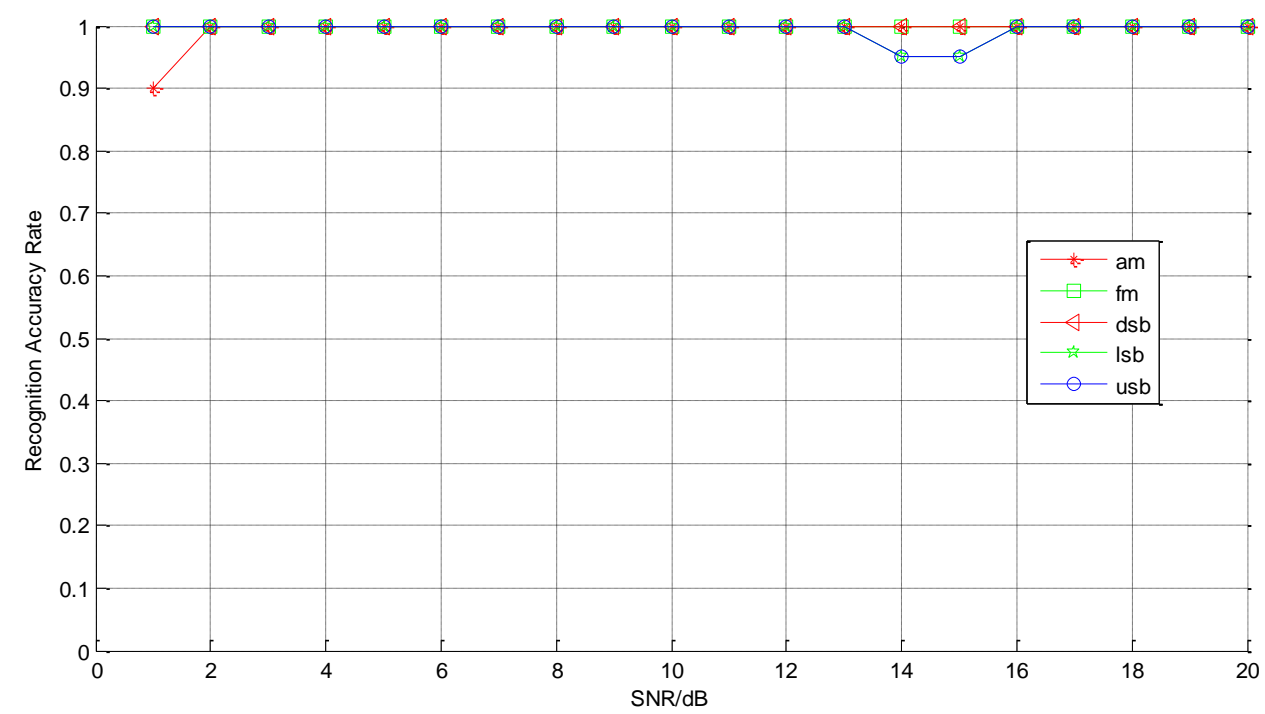

Figure 10. Accuracy Rate of Analog Modulation Signals Recognition

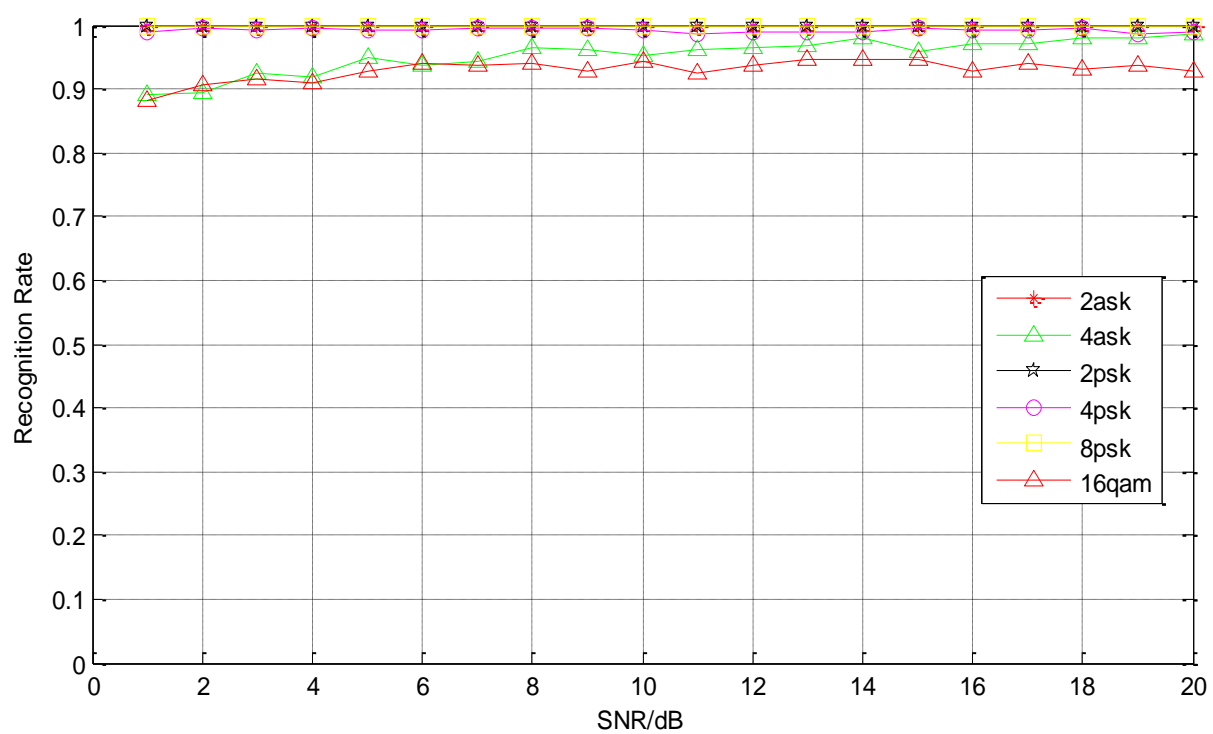

Figure 11. Accuracy Rate of Digital Modulation Signals Recognition

As shown in Figure 10 and Figure 11, the parameter $J$ and high-order cumulants have a very good performance in noise resistance ability. The Recognition rate remains as high as $90 \%$ above with the change of SNR. 


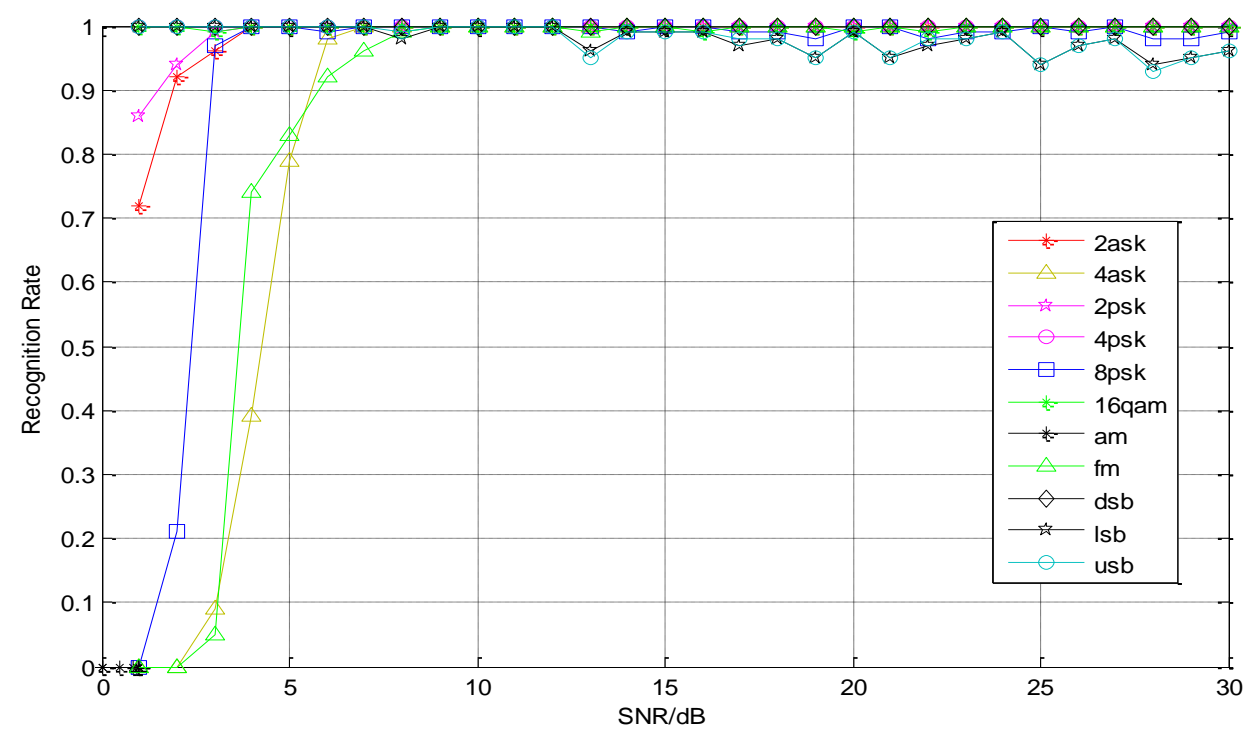

Figure 12. Accuracy Rate of all Reference Signals Recognition

It is clear that the recognition algorithm has a good recognition accuracy performance of greater than $90 \%$ when $S N R \geq 6 \mathrm{~dB}$. The performance of the algorithm in this paper is much better than others and meanwhile the computational complexity is not high which can satisfy the real-time requirements.

Here is the recognition rate of the reference signals when SNR equals to 10dB in Table 4 below.

Table 4. Recognition Rate of Reference Signals

\begin{tabular}{|c|c|c|c|c|c|c|c|c|c|c|}
\hline & AM & FM & DSB & LSB & USB & $\begin{array}{c}\text { 2ASK/B } \\
\text { PSK }\end{array}$ & 4ASK & QPSK & 8PSK & $\begin{array}{c}\text { 16QA } \\
\text { M }\end{array}$ \\
\hline AM & 0.9200 & 0.0200 & 0 & 0 & 0 & 0.0600 & 0 & 0 & 0 & 0 \\
\hline FM & 0.0050 & 0.9950 & 0 & 0 & 0 & 0 & 0 & 0 & 0 & 0 \\
\hline DSB & 0 & 0 & 1 & 0 & 0 & 0 & 0 & 0 & 0 & 0 \\
\hline LSB & 0 & 0 & 0 & 0.9450 & 0.0550 & 0 & 0 & 0 & 0 & 0 \\
\hline USB & 0 & 0 & 0 & 0.0150 & 0.9850 & 0 & 0 & 0 & 0 & 0 \\
\hline $\begin{array}{c}\text { 2ASK/ } \\
\text { BPSK }\end{array}$ & 0 & 0 & 0.0300 & 0 & 0 & 0.9280 & 0.0420 & 0 & 0 & 0 \\
\hline 4ASK & 0.0700 & 0 & 0 & 0 & 0 & 0.0200 & 0.9100 & 0 & 0 & 0 \\
\hline QPSK & 0 & 0 & 0 & 0 & 0 & 0 & 0 & 0.9930 & 0.0070 & 0 \\
\hline 8PSK & 0 & 0 & 0 & 0 & 0 & 0 & 0 & 0 & 1 & 0 \\
\hline 16QAM & 0 & 0 & 0 & 0 & 0 & 0.0100 & 0.0010 & 0.0520 & 0.0180 & 0.9190 \\
\hline
\end{tabular}

\section{Conclusion}

Communication signal's recognition is an important problem for the electronic countermeasure procedure. In this paper, the method how to classify communication signals including AM, FM, DSB, LSB, USB, 2ASK/BPSK, 4ASK, QPSK, 8PSK, 16QAM is provided. Aiming at the problem of low recognition rate of recognition between analog modulation and digital modulation signals in low SNR, a improved 
method is given, which solves the carrier blind point problem and scale selection difficulty problem by down converting the signals to baseband. For the recognition approaches of analog and digital modulation signal, the excellent noise-resistance parameters $J$ and high-order cumulants are introduced. The final result shows that the method has a better recognition result and has a well prospect in communication signal's recognition.

\section{Acknowledgment}

This work was supported by the Key Development Program of Basic Research of China(JCKY2013604B001), the Nation Nature Science Foundation of China (61301095), Nature Science Foundation of Heilongjiang Province of China (F201408) and the Fundamental Research Funds for the Central Universities (No. HEUCF100814 and HEUCF100816).

Meantime, all the authors declare that there is no conflict of interests regarding the publication of this article.

\section{References}

[1] Y. T. Chan, J. W. Plews and K. C. Ho, "Symbol rate estimation by the wavelet transform", Circuits and Systems, 1997. ISCAS '97, Proceedings of 1997 IEEE International Symposium on. IEEE, vol. 1, (1997).

[2] K. C. Ho, W. Prokopiw and Y. T. Chan, "Modulation identification of digital signals by the wavelet transform”, IEE Proceedings-Radar, Sonar and Navigation, vol. 4, no. 147, (2000).

[3] X. Jun, "The study of some issues of short data processing in communication blind receiving", Qinghua University, (2007).

[4] W. Jin, Z. Yili and X. Jianfang, "An analog signal modulation identification method under low SNR", Shanxi Electronic Technology, vol. 1, (2008).

[5] A. K. Nandi and E. E. Azzouz, "Automatic analogue modulation recognition”, Signal Processing, vol. 2, no. $46,(\mathbf{1 9 9 5})$.

[6] A. Swami and B. M. Sadler, "Hierarchical digital modulation classification using cumulants", Communications IEEE Transactions, vol. 3, no. 48, (2000).

[7] V. D. Orlic and M. L. Dukic, "Automatic modulation classification: Sixth-order cumulants features as a solution for real-world challenges", Telecommunications Forum (TELFOR), $201220^{\text {th }}$, IEEE, (2012).

[8] C. Weidong and Y. Shaoquan, "Classification of MPSK signal based on cumulants", Journal of electronic science and technology of xi 'an university: Natural Science Edition, vol. 2, no. 29, (2004).

[9] Y. T. Chan, J. W. Plews and K. C. Ho, "Symbol Rate Estimation by the Wavelet Transform", ISCAS '97, vol. 1, (1997).

[10] H. Yanping and L. Guangsen, "Automatically identify digital modulation signals By using the statistical parameters", Journal of communication, vol. 2, no. 23, (2002). 
International Journal of Future Generation Communication and Networking Vol. 10, No. 1 (2017) 